\title{
Effects of auditory reliability and ambiguous visual stimuli on auditory spatial discrimination
}

Madeline S. Cappelloni ${ }^{1,2,3}$, Sabyasachi Shivkumar ${ }^{3,4}$, Ralf M. Haefner ${ }^{3,4}$, Ross K. Maddox ${ }^{1,2,3,5}$

${ }^{1}$ Biomedical Engineering, University of Rochester, Rochester, NY 14627, USA

${ }^{2}$ Del Monte Institute for Neuroscience, University of Rochester, Rochester, NY 14627, USA

${ }^{3}$ Center for Visual Science, University of Rochester, Rochester, NY 14627, USA

${ }^{4}$ Brain and Cognitive Sciences, University of Rochester, Rochester, NY 14627, USA

${ }^{5}$ Neuroscience, University of Rochester, Rochester, NY 14627, USA 


\section{ABSTRACT}

The brain combines information from multiple sensory modalities to interpret the environment.

Multisensory integration is often modeled by ideal Bayesian causal inference, a model proposing that perceptual decisions arise from a statistical weighting of information from each sensory modality based on its reliability and relevance to the observer's task. However, ideal Bayesian causal inference fails to describe human behavior in a simultaneous auditory spatial discrimination task in which spatially aligned visual stimuli improve performance despite providing no information about the correct response. This work tests the hypothesis that humans weight auditory and visual information in this task based on their relative reliabilities, even though the visual stimuli are task-uninformative, carrying no information about the correct response, and should be given zero weight. Listeners perform an auditory spatial discrimination task with relative reliabilities modulated by the stimulus durations. By comparing conditions in which task-uninformative visual stimuli are spatially aligned with auditory stimuli or centrally located (control condition), listeners are shown to have a larger multisensory effect when their auditory thresholds are worse. Even in cases in which visual stimuli are not task-informative, the brain combines sensory information that is scene-relevant, especially when the task is difficult due to unreliable auditory information. 


\section{I. INTRODUCTION}

When we navigate our surroundings, we encounter sensory information from multiple

3 modalities. Combining complementary information across sensory modalities often helps us

4 construct a more accurate percept of the world. In contrast, combining conflicting or irrelevant

5 sensory information can lead to perceptual errors. In order to optimize perceptual accuracy, the

6 brain must determine whether to combine information across sensory modalities, and if so, how to

7 weigh each sensory modality. Formally, the notion of reliability weighting is described by Bayesian

8 models of cue combination - forced integration (Ernst and Banks, 2002) and more recently causal

9 inference (Körding et al., 2007). In these models, each cue is treated as a measurement of the

10 stimulus with a Gaussian distribution of the likelihood of the stimulus based on that measurement.

11 The multisensory measurement is then a combination of unisensory measurements weighted by the

12 inverse of their relative variances, such that a narrower likelihood distribution will have more

13 influence on the combined percept. Importantly, the causal inference model adds another layer of

14 inference to this model, in which the degree of cue integration depends on the probability that both

15 measurements actually arose from the same event in the world (Körding et al., 2007). 
17 can use information from multiple modalities to determine the correct response; for example, an

18 audiovisual localization task in which the subject is asked where a noise and light occurred (Körding

19 et al., 2007). Under good visual conditions, this task gives rise to the "ventriloquist effect", a bias of

20 auditory location towards the visual stimulus (Howard and Templeton, 1966). However, when the

21 visual stimulus gets blurrier and harder to localize relative to the auditory stimulus, the apparent

22 visual bias weakens or even manifests as an auditory bias of perceived visual location (Alais and

23 Burr, 2004). This demonstrates that the ventriloquist effect is truly a bias of both visual and auditory

24 stimuli towards each other with the magnitude of the bias determined by the relative reliability of

25 each modality. Importantly, in this and other tasks described by the model, there is only one

26 stimulus in each sensory modality, both of which are informative about the correct response. In this

27 scenario, it is optimal for the brain to use multisensory integration to improve its judgment and

28 behavioral performance. 
30 cues and found a multisensory effect of visual stimuli on auditory spatial processing (Cappelloni et

31 al., 2019) even when those visual cue did not contain any task-relevant information. We asked

32 listeners to perform a concurrent auditory spatial discrimination task in which random visual stimuli

33 were either spatially aligned with two symmetrically separated auditory stimuli or both collocated in

34 the center of the screen, and found a performance benefit when the visual stimuli were spatially

35 aligned. This audiovisual effect goes against the traditional conception of multisensory integration as

36 a mechanism for the optimal combination of information from the environment (Ernst and

37 Bülthoff, 2004). The benefit provided by the spatially aligned visual stimuli is also not explained by

38 an ideal Bayesian observer (whose response should be invariant to the locations of the task-

39 uninformative visual stimuli), and is counterintuitive in that the visual stimuli do not help to

40 determine the correct response and must instead benefit the listener through process not part of the

41 ideal Bayesian observer model.

42 Here we test the hypothesis that the brain weighs auditory and visual stimuli by their relative

43 reliabilities even in the case where the visual stimuli do not provide any information about the

44 correct response and would be ignored by an ideal observer. We modulated the reliability of the

45 auditory stimuli by changing their duration, with longer auditory stimuli being more reliable. We

46 found that the benefit provided by the visual stimuli is larger where subjects had poor auditory

47 thresholds. Our results replicate those of our previous study (Cappelloni et al., 2019) and further

48 investigate the ways in which scene-relevant but task-uninformative stimuli can shape perception

49 providing constraints for future theoretical models.

II. METHODS

51 A. Participants 
Participants (16 female, 4 male; ages ranging between 18 and 31, mean 21.5 +/- 3 years) with

53 normal hearing (thresholds $20 \mathrm{~dB}$ HL or better at 500-8000 Hz) and normal vision (self-reported)

54 gave written informed consent. They were compensated for the full duration of time spent in the

55 lab. Research was performed in accordance with protocol approved by the University of Rochester

56 Research Subjects Review Board.

57 B. Stimuli

58 Auditory stimuli were pink noise tokens and harmonic tone complexes with matching

59 spectral envelopes bandlimited to $220-4000 \mathrm{~Hz}$. Stimuli were generated and localized by HRTFs

60 from the CIPIC library using interpolation from python's expyfun library as in (Cappelloni et al.,

61 2019), with the notable difference that we generated the pink noise tokens and harmonic tone

62 complexes to be three durations, $100 \mathrm{~ms}, 300 \mathrm{~ms}$, and $1 \mathrm{~s}$. Auditory stimuli were presented at a

$6324414 \mathrm{~Hz}$ sampling frequency and $65 \mathrm{~dB}$ SPL level from TDT hardware (Tucker Davis

64 Technologies, Alachua, FL) over ER-2 insert earphones (Etymotic Research, Elk Grove Village, IL).

65 Visual stimuli were regular polygons of per-trial random number of sizes and color. They

66 were inscribed within a $1.5^{\circ}$ diameter circle. Colors were chosen to have uniform saturation and

67 luminance, with the two stimuli in each trial having opposite hue as in (Cappelloni et al., 2019).

68 Visual stimuli had the same onset and offset times as the auditory stimuli and thus matched their

69 duration. To prevent overlap they were presented in alternating frames (Blaser et al., 2000) on a

70 monitor with a $144 \mathrm{~Hz}$ refresh rate.

71 C. Task 
Each trial began when the subject fixated on a white dot in the center of the screen,

73 confirmed with an eye tracking system (EyeLink 1000, SR Research). Then all four auditory and

74 visual stimuli were presented concurrently for the duration of the trial (100 ms, $300 \mathrm{~ms}$, or $1000 \mathrm{~ms})$.

75 After stimulus presentation, subjects were asked to respond with what side the tone was on by

76 pressing a button. There were two visual conditions: one in which the visual stimuli were spatially

77 aligned with the auditory stimuli and one in which the visual stimuli were collocated in the center of

78 the screen.

79 We presented trials according to weighted one up one down adaptive tracks that adjusted the

80 separation of the two sounds (Kaernbach, 1991). Separations were adjusted on a log scale such that

81 separation increased by a factor of 2 when the participant responded incorrectly and decreased by a

82 factor of $\mathbf{2}^{\mathbf{1 / 3}}$ when they responded correctly. Each track had 130 trials and began at a starting

83 separation of $10^{\circ}$ azimuth. For each track, we randomized the number of trials with the tone on the

84 left and right. There were six tracks, three durations by two visual conditions, that were randomly

85 interleaved.

86 D. Analysis

87 In order to obtain $75 \%$ thresholds we averaged the level at each reversal (skipping the first six

88 reversals). Threshold improvement is defined as the difference between the separation thresholds of

89 the two visual conditions (central - matched). We resampled the reversals with replacement to

90 determine the significance of each threshold improvement (positive or negative respectively - less

91 than $2.5 \%$ or greater than $97.5 \%$ of resampled threshold improvements less than zero). We

92 performed linear regression on the central threshold vs. threshold improvement data and computed

$9395 \%$ confidence intervals using the Python seaborn package (Michael Waskom et al., 2017). We also

94 fit a linear mixed effects model to the data using Python's statsmodels package (Seabold and

95 Perktold, 2010). We fit the thresholds with a random intercept model such that each subject is 
bioRxiv preprint doi: https://doi.org/10.1101/2020.06.11.140491; this version posted June 12, 2020. The copyright holder for this preprint (which was not certified by peer review) is the author/funder, who has granted bioRxiv a license to display the preprint in perpetuity. It is made available under aCC-BY-NC-ND 4.0 International license.

96 assigned an intercept to control for between subject variance. We considered categorical visual

97 condition, duration, and interaction of visual condition and duration as fixed effects in the model. 


\section{III. RESULTS}
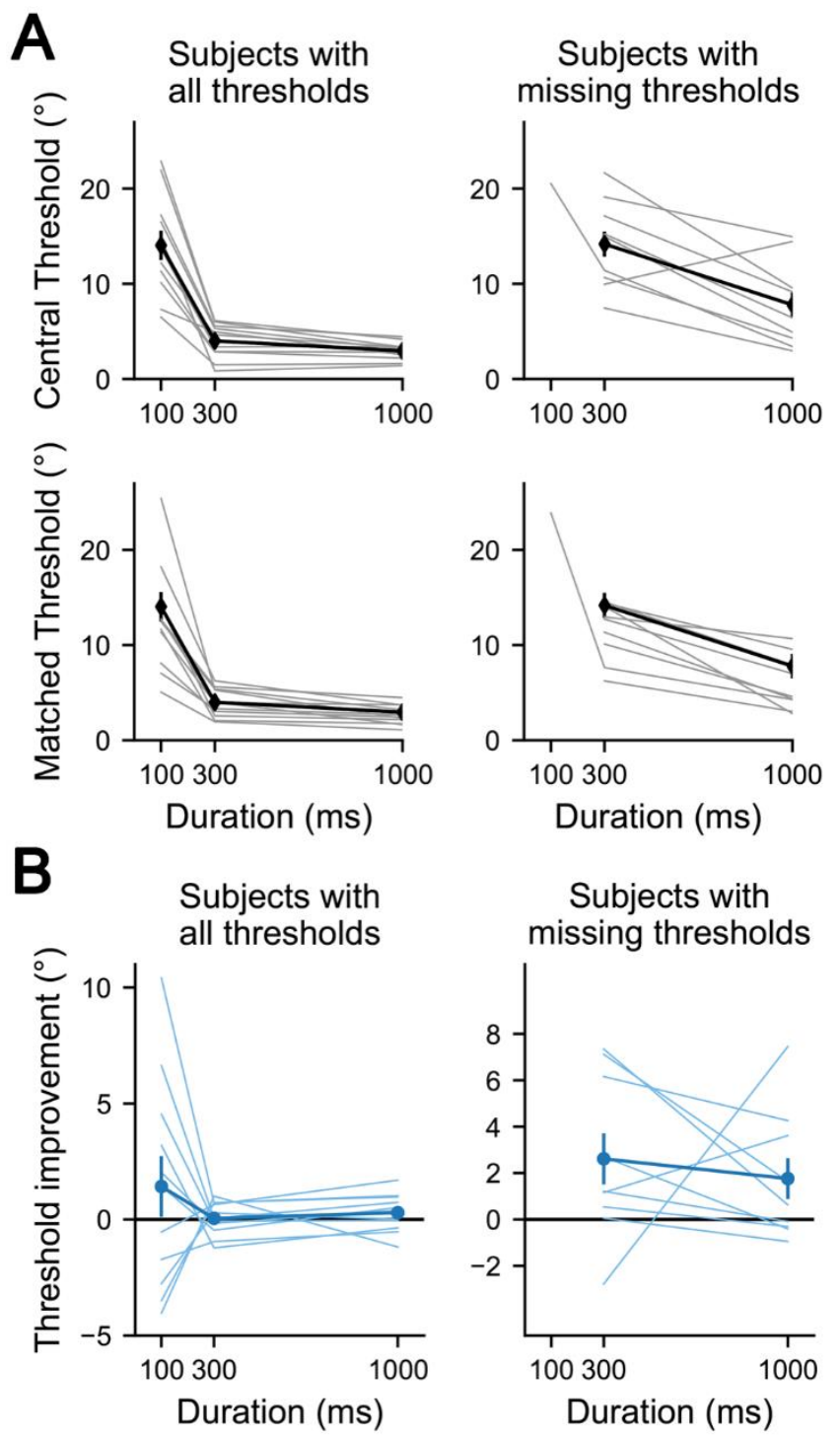

99

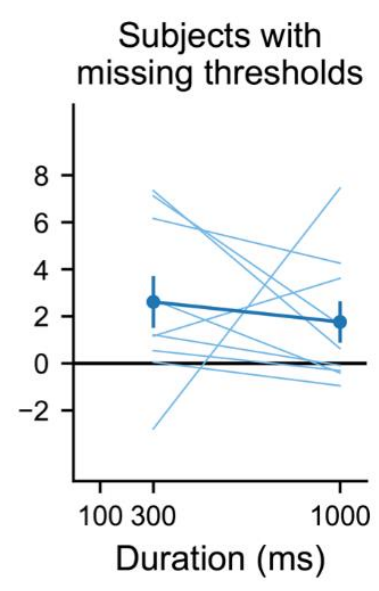

100 Fig. 1. (Color Online). A. Thresholds for each duration in the central visual condition (top) and

101 matched visual condition (bottom). Many subjects had missing thresholds (too large to measure

102 accurately) in one or both visual conditions at $100 \mathrm{~ms}$ and are plotted in the right column $(\mathrm{n}=9)$

103 while the remainder are plotted on the left $(n=11)$. B. Improvements in threshold for the two groups

104 of subjects: those who could perform the task at all durations (left), those had one or both

105 thresholds missing at $100 \mathrm{~ms}$ (right). 
107 asymptotically in both visual conditions as the duration of the auditory stimuli increased; however,

108 there was considerable variation in subject performance. Only 11 of 20 subjects were able to

109 perform the auditory discrimination task at the shortest duration such that we could calculate a

110 separation threshold (Figure 1A). Subjects in this group had a large decrease in threshold between

$111100 \mathrm{~ms}$ and $300 \mathrm{~ms}$, but did not improve further for $1000 \mathrm{~ms}$ stimuli. For the remainder of the

112 subjects who had thresholds too big to calculate in either or both $100 \mathrm{~ms}$ conditions, they improved

113 their threshold between $300 \mathrm{~ms}$ and $1000 \mathrm{~ms}$. In a linear mixed effect model of all subjects

114 combined, only duration $\left(\mathrm{p}=9.5 \times 10^{-9}\right)$ had a significant effect on threshold. Visual condition and the

115 interaction of visual condition and duration did not have a significant effect on threshold.

116 We defined "threshold improvement" as the difference between the central and matched

117 visual conditions and used it to measure the size of the visual benefit (Figure 1B). Differences in

118 individual auditory spatial processing ability indicate that auditory reliability was not uniform within

119 a given duration condition. 
A

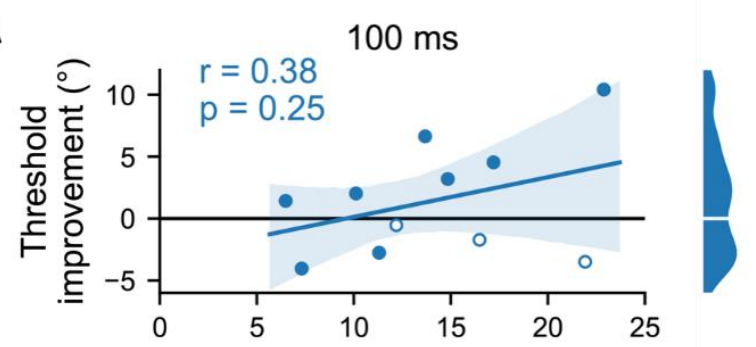

B

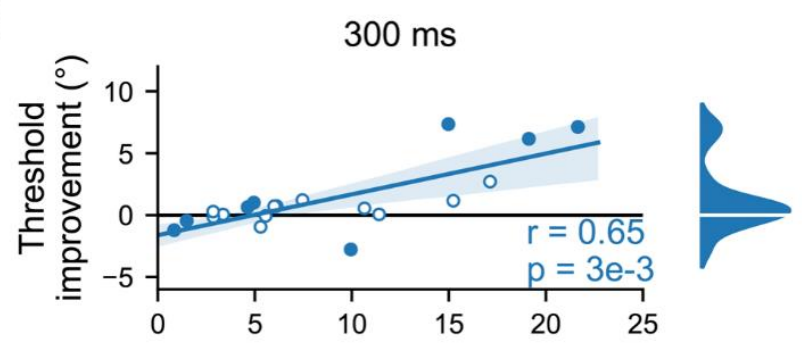

C

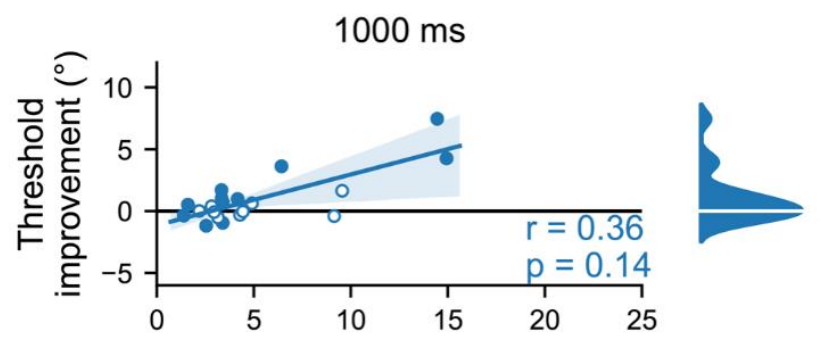

D

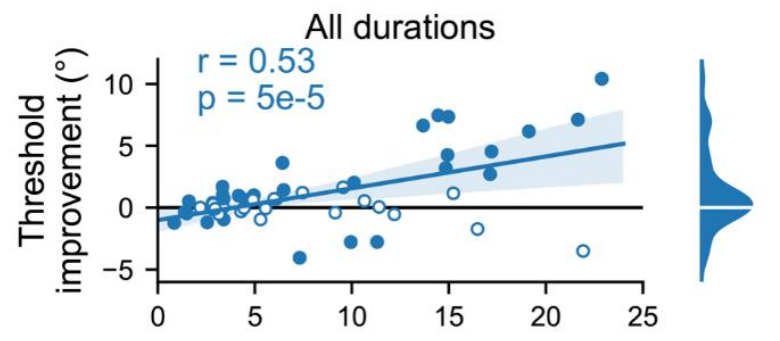

E

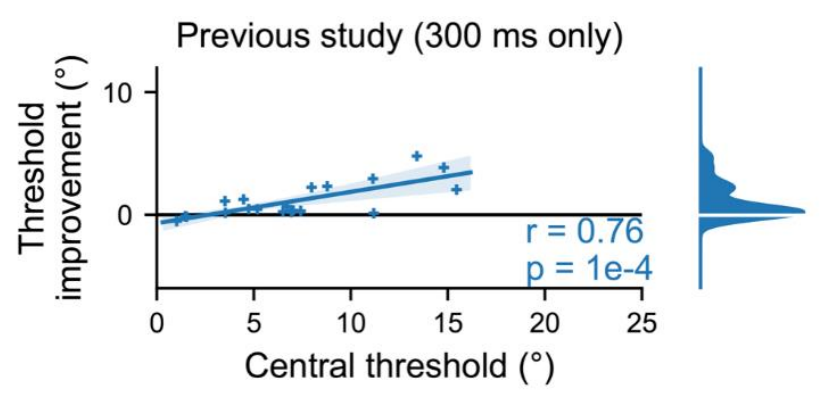

121 
122 Fig. 2. (Color Online). Linear regression of threshold improvements against central threshold. Solid

123 markers indicated significant differences between the two visual conditions based on within subject

124 variation $(\boldsymbol{\alpha}=\mathbf{0 . 0 5}$, uncorrected). Open markers indicate no significant effect of visual stimuli.

125 Also shown are marginal kernel density estimates (excluding tails beyond which there is less than

$1260.5 \%$ of the mass). A-C. Separate regressions for each duration condition. D. Regression for

127 threshold improvements regardless of duration. E. Regression of data from our previous study

128 (Cappelloni et al., 2019).

In order to compensate for individual differences we used the separation threshold in the

130 central condition as a measure of auditory reliability for each duration condition (Figure 2). Larger

131 thresholds indicated that the task was more difficult and the individual's auditory reliability was likely

132 worse. Pooling measurements across durations, we found a linear relationship between the threshold

133 improvement and central threshold (Figure $2 \mathrm{D}, \mathrm{r}=0.53, \mathrm{p}=5 \times 10^{-5}$ ). A similar trend is shown when

134 plotting data from our previous study (Cappelloni et al., 2019) (only using $300 \mathrm{~ms}$ stimuli) on the

135 same axes (Figure 2E, $\mathrm{r}=0.76, \mathrm{p}=10^{-4}$ ).

\section{IV. DISCUSSION}

137 We found that performance in the auditory task correlated with the benefit subjects receive from

138 task-uninformative visual stimuli. Listeners experienced the most benefit when the auditory task was

139 difficult for them (large central threshold). In contrast, individuals who did well in the auditory task

140 (small central threshold) experienced no benefit or even a slight decrement.

141 It should be noted that as the central threshold gets larger, the stimuli become more peripheral

142 where spatial acuity is worse (Hafter and Maio, 1998; Maddox et al., 2014; Middlebrooks and Onsan,

143 2012; Mills, 1958), compounding listeners’ worse auditory reliability. A similar decrease may also

144 occur in visual location reliability. Extending the duration improves listener's thresholds in both 
145 visual conditions, which can be explained by an improvement in the auditory reliability, suggesting

146 that our duration manipulation does roughly correlate with reliability.

147 This work replicates our previous finding that task uninformative but spatially aligned stimuli

148 benefit auditory spatial discrimination. In the previous study, the visual stimuli preceded auditory

149 stimuli by $100 \mathrm{~ms}$, whereas in this study, their onsets were all concurrent. Additionally, we previously

150 used sigmoidal fits to determine threshold instead of adaptive tracks. This suggests that the visual

151 benefit is robust to small audiovisual asynchronies and changes in probabilistic distribution of

152 stimuli across space (adaptive tracks will lead to more non-uniform priors).

153 Although the visual benefit was larger where subjects showed worse auditory performance, and

154 duration had a significant effect on task difficulty, we did not see a significant effect of changing the

155 duration on visual benefit. This is mainly due to the wide range of auditory spatial processing

156 abilities among the subjects. Because of differences in auditory spatial processing, the effect of

157 duration on visual benefit was inconsistent across subjects. Subjects could be divided roughly into

158 two groups with two different patterns of thresholds, those who could reliably perform the task at

$159100 \mathrm{~ms}$ and those who could not. The former group improved their performance significantly when

160 the duration was extended to $300 \mathrm{~ms}$, but did not further improve when the stimuli were $1000 \mathrm{~ms}$,

161 suggesting that they reached ceiling performance at $300 \mathrm{~ms}$. In contrast, the latter group improved

162 significantly when the stimulus duration extended from $300 \mathrm{~ms}$ to $1000 \mathrm{~ms}$ and were not at ceiling

163 performance at $300 \mathrm{~ms}$. In both this study and our original experiment (Cappelloni et al., 2019),

164 which only included the 300 ms duration condition, we observed a wide range of auditory

165 thresholds. In addition to simple variability across subjects, some thresholds were missing data

166 points because the monitor on which visual stimuli were displayed could not extend far enough to

167 accurately measure large thresholds. These missing data may have allowed us to better fit a model

168 that could show an effect of changing the stimulus duration on visual benefit if such an effect 
169 existed. Without considering effects on the scale of individual subjects, for which we did not have

170 enough data, we could not establish a causal relationship between changing stimulus duration and

171 the visual benefit even though correlations suggest one may exist.

172 It is possible that auditory reliability is the underlying factor driving the relationship between

173 auditory performance and visual benefit, even though our data did not show a clear relationship

174 between duration and visual benefit. If auditory reliability does modulate the effect of task-

175 uninformative visual stimuli, following the spirit of Bayesian causal inference, it would further

176 violate the central assumption that the brain will only integrate information that is relevant to the

177 task. This would point to a broader notion of multisensory perception in which stimuli are

178 integrated based on their reliability in representing the sensory scene, rather than the reliability of

179 information they provide regarding a specific task. Further work is needed to describe the

180 boundaries of what information is integrated in a scene. It is important that such work go beyond

181 traditional paradigms to those that can reveal differences of scene-relevant vs task-informative cues

182 V. CONCLUSION

183 We show that listeners gain a larger benefit from task-uninformative visual stimuli in an auditory

184 spatial discrimination task when the auditory task is difficult. Our results are consistent with, but do

185 not confirm, the notion that reliability weighting as described in Bayesian models may occur even

186 when visual stimuli do not carry information about the correct decision in the task. We believe two

187 next steps would clarify the findings of this paper. "Small-n" design in which few subjects are

188 recruited to complete many trials would allow us to understand perception on the level of

189 individuals, rather than generalizing across a diverse population (Smith and Little, 2018). Secondly,

190 we call for an exploration of more complex paradigms with multiple multimodal cues caused by

191 potentially multiple events in the world that provide new and stronger tests of existing models.

\section{$192 \quad$ ACKNOWLEDGMENTS}


The authors wish to acknowledge Sara Fiscella for assisting with data collection.

196 Other Communication Disorders of the National Institutes of Health under award number

197 R00DC014288.

\section{REFERENCES}

Alais, D., and Burr, D. (2004). “The Ventriloquist Effect Results from Near-Optimal Bimodal Integration,” Curr. Biol., 14, 257-262. doi:10.1016/j.cub.2004.01.029 space," Nature, 408, 196-.

203 Cappelloni, M. S., Shivkumar, S., Haefner, R. M., and Maddox, R. K. (2019). "Task-uninformative visual stimuli improve auditory spatial discrimination in humans but not the ideal observer,"

206 Ernst, M. O., and Banks, M. S. (2002). "Humans integrate visual and haptic information in a statistically optimal fashion," Nature, 415, 429-433. doi:10.1038/415429a

Ernst, M. O., and Bülthoff, H. H. (2004). "Merging the senses into a robust percept," Trends Cogn. Sci., 8, 162-169. doi:10.1016/j.tics.2004.02.002

210 Hafter, E. R., and Maio, J. D. (1998). “Difference thresholds for interaural delay,” J. Acoust. Soc.

212 Howard, I. P., and Templeton, W. B. (1966). Human spatial orientation, Human spatial orientation, $213 \quad$ John Wiley \& Sons, Oxford, England, 533 pages.

214 Kaernbach, C. (1991). "Simple adaptive testing with the weighted updown method," Percept. 
216 Körding, K. P., Beierholm, U., Ma, W. J., Quartz, S., Tenenbaum, J. B., and Shams, L. (2007).

217 "Causal Inference in Multisensory Perception," PLOS ONE, 2, e943.

218 doi:10.1371/journal.pone.0000943

219 Maddox, R. K., Pospisil, D. A., Stecker, G. C., and Lee, A. K. C. (2014). “Directing Eye Gaze

220 Enhances Auditory Spatial Cue Discrimination,” Curr. Biol., 24, 748-752.

221 doi:10.1016/j.cub.2014.02.021

222 Michael Waskom, Olga Botvinnik, Drew O’Kane, Paul Hobson, Saulius Lukauskas, David C

223 Gemperline, Tom Augspurger, et al. (2017). mwaskom/seaborn: v0.8.1 (September 2017),

$224 \quad$ Zenodo. doi:10.5281/zenodo.883859

225 Middlebrooks, J. C., and Onsan, Z. A. (2012). “Stream segregation with high spatial acuity,” J.

226 Acoust. Soc. Am., 132, 3896-3911. doi:10.1121/1.4764879

227 Mills, A. W. (1958). “On the Minimum Audible Angle,” J. Acoust. Soc. Am., 30, 237-246.

$228 \quad$ doi:10.1121/1.1909553

229 Seabold, S., and Perktold, J. (2010). "Statsmodels: Econometric and Statistical Modeling with

230 Python," Austin, Texas, 92-96. Presented at the Python in Science Conference.

231 doi:10.25080/Majora-92bf1922-011

232 Smith, P. L., and Little, D. R. (2018). "Small is beautiful: In defense of the small-N design," Psychon.

233 Bull. Rev., , doi: 10.3758/s13423-018-1451-8. doi:10.3758/s13423-018-1451-8 
Subjects with all thresholds
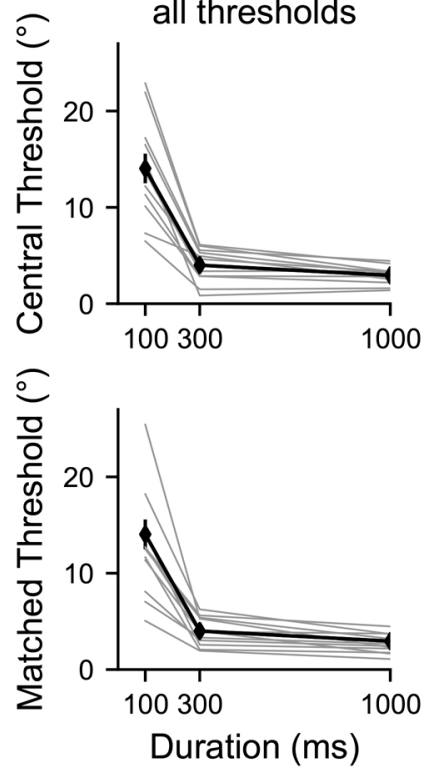

Subjects with all thresholds

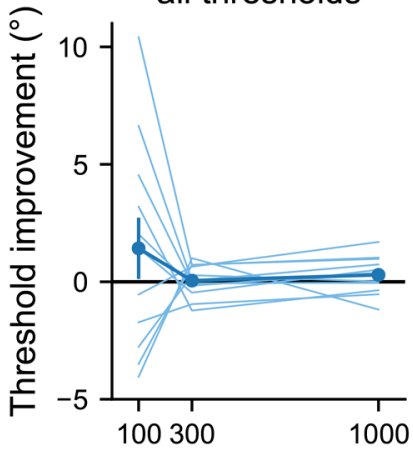

Duration (ms)
Subjects with missing thresholds
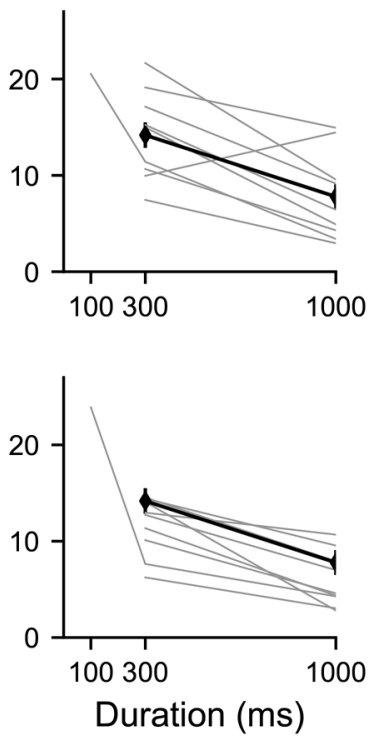

Subjects with missing thresholds

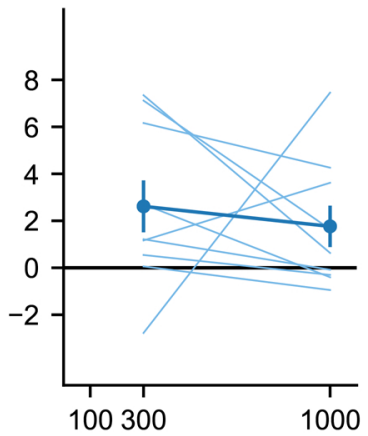

Duration (ms) 


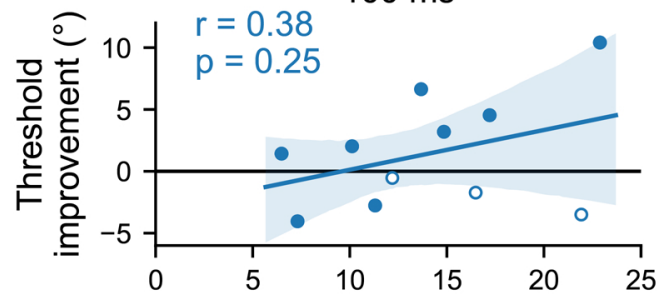

B

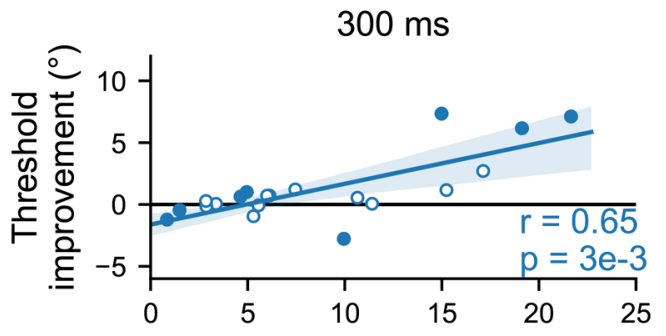

C

$1000 \mathrm{~ms}$
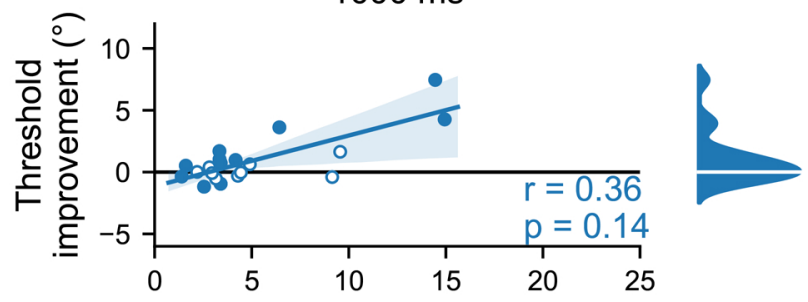

D
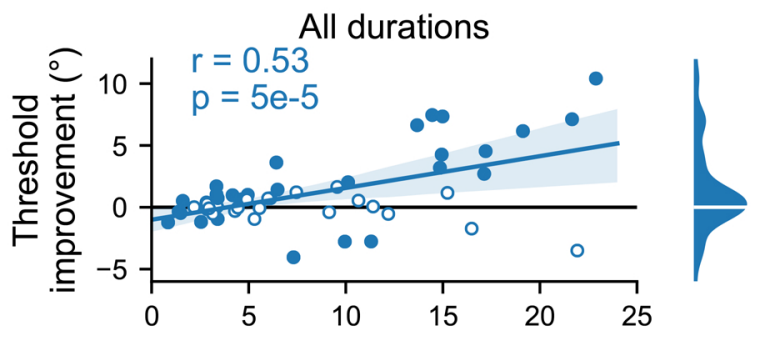

E

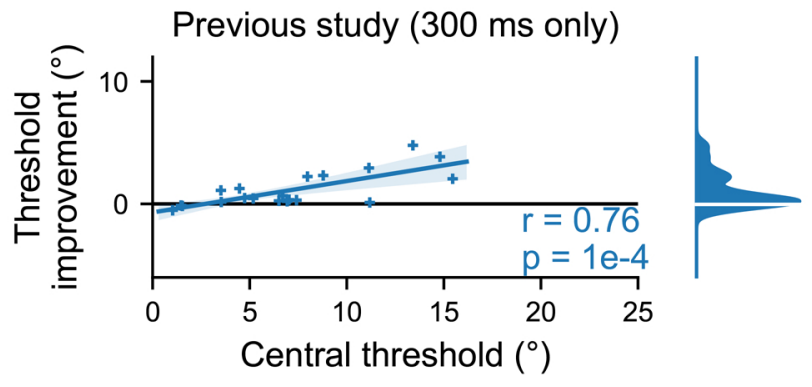

